\title{
La gestión estratégica de la responsabilidad social en las pyme venezolanas
}

Strategic Management of Social Responsibility in Venezuelan SMEs

\section{A gestão estratégica da responsabilidade social nas MPE venezuelanas}

\section{José Antonio Martínez Velasco*}

Fecha de recibido: 26 de enero de 2015

Fecha de aprobado: 5 de agosto de 2015

Doi: dx.doi.org/10.12804/rev.univ.empresa.30.2016.10

Para citar este artículo: Martínez Velasco, J. A. (2016). La gestión estratégica de la responsabilidad social en las pyme venezolanas. Universidad \& Empresa, 18(30), 191-209. Doi: dx.doi.org/10.12804/rev.univ. empresa.30.2016.10

\section{RESUMEN}

Como unidades económico-productivas con características propias, las pyme han evolucionado hasta convertirse en la categoría de organizaciones con mayor impacto económico y social. Aunque existen diversos campos en las Ciencias Administrativas relacionados con la gestión de este tipo de empresas, el de la gestión estratégica ha logrado consolidarse como el más propicio para el logro de la competitividad. La responsabilidad social empresarial (RSE) ha emergido como una práctica común en las grandes empresas, que hoy es implementada en casi todo el planeta, incluso en las pyme, por iniciativa propia o por mandato legal. De acuerdo con los resultados analizados desde el método hermenéutico, a partir de una investigación cualitativa soportada en las técnicas de la observación participante y la entrevista en profundidad, en este artículo se expone una alternativa para

Doctor en Ciencias Administrativas y Gerenciales, de la Universidad de Carabobo (Venezuela). Profesor en el programa de Doctorado en Ciencias Administrativas y Gerenciales, de la Universidad de Carabobo y miembro del Centro de investigación y desarrollo de la pequeña y mediana empresa y la microempresa del estado Carabobo (Venezuela). Correo electrónico: venlider@hotmail.com 
el abordaje de la RSE por parte de los directivos de estas organizaciones en el marco de la gestión estratégica.

Palabras clave: empresa, empresario, gestión estratégica, pyme, responsabilidad social empresarial (RSE).

\section{ABSTRACT}

SMEs, as productive economic units with their own characteristics, have evolved into the category of companies with greater economic and social impact. Although there are various work fields in management sciences related to the management of SMEs, the field of strategic management has established itself as the most conducive to achieving competitiveness in these organizations. Corporate Social Responsibility (CSR) has emerged as a common practice for large companies, but currently is implemented in most of the world, even into small and medium enterprises. This, either voluntarily or by legal mandate. According to the results analyzed following the hermeneutical method, parting from a qualitative research based on the techniques of participant observation and in-depth interviews, in this article an alternative for addressing corporate social responsibility by managers of this kind of organizations is presented, in accordance with the strategic management framework.

Keywords: Company, entrepreneur, strategic management, SME, corporate social responsibility (CSR).

\section{RESUMO}

As MPE, como unidades económico-produtivas com características próprias, têm evolucionado até converter-se talvez na categoria de organizações com maior impacto económico e social. Ainda que existem diversos campos nas ciências administrativas relacionados com a gestão deste tipo de empresas, o da gestão estratégica tem conseguido consolidar-se provavelmente como o mais propício para o sucesso da competitividade nas mesmas. A responsabilidade social empresarial (RSE) tem emergido como uma prática comum nas grandes empresas, mas atualmente resulta implementada em quase o planeta todo, inclusive nas MPE, isto por iniciativa própria ou por mandato legal. De acordo com os resultados analisados segundo o método hermenêutico, a partir de uma pesquisa qualitativa suportada nas técnicas da observação participante e a entrevista em profundidade, neste artigo se expõe uma alternativa para a abordagem da SER por parte dos diretivos deste tipo de organizações, de acordo com o marco da gestão estratégica.

Palavras-chave: empresa, empresário, gestão estratégica, MPE, responsabilidade social empresarial (RSE). 


\section{INTRODUCCIÓN}

De acuerdo con el discurso en las Ciencias Administrativas y Gerenciales, la responsabilidad social empresarial (RSE) es un concepto en desarrollo que se expresa de diversos modos, no solo por parte del gran empresario, sino también del gerente de las pequeñas y medianas empresas (pyme), quien está interesado en la continuidad del negocio, en armonía con sus stakeholders y la sociedad. El gerente de las pyme, dispuesto a dirigir su negocio de manera socialmente responsable, puede recurrir a técnicas y métodos fundamentados en su creatividad y, entre estas, puede basarse también en la estrategia, como se plantea en este texto.

El presente artículo ha sido estructurado en tres secciones. Estas no solo destacan el concepto y las implicaciones de la RSE, sino que también exponen las consideraciones esenciales relacionadas con las pyme venezolanas y su gestión estratégica.

En la primera sección se individualiza la pequeña y mediana empresa como un ente socioproductivo con características propias y distintivas de las otras categorías de organizaciones. Asimismo, se presentan los aspectos clave para la identificación de las pyme venezolanas y sus procesos básicos de gestión. En la segunda se analiza la gestión estratégica de las empresas en general y también en función de la pequeña y mediana empresa. Se establecen las diferencias entre este tipo de compañías y las grandes empresas, con el fin de resaltar la estrategia como una herramienta decisiva en el entorno global. El tercer aparte del texto muestra la RSE como una acción común del empresario en la gestión de su negocio, ya sea grande o del tipo pyme. En esta sección también se discuten las posibilidades para la pequeña y mediana empresa venezolana, a propósito de la implementación de una gestión socialmente responsable.

\section{MARCO REFERENCIAL}

\subsection{Las pyme en la República Bolivariana de Venezuela}

Las primeras referencias de entes organizativos que es posible considerar como pyme se remontan a la Edad Media (Martín Martín \& Cepeda González, 2006). En aquella época, las pyme correspondían a asociaciones comerciales compuestas por un limitado número de individuos ligados por parentescos. Estas trataban de satisfacer desde las necesidades más elementales hasta las más sofisticadas de sus clientes, mediante una ejecución artesanal que imprimía a sus encargos una 
verdadera maestría. Sus integrantes eran capacitados mediante un método informal, impartido por el predecesor (maestro) para el sucesor (aprendiz) en el seno de la propia familia.

Como en la mayoría de las Naciones, las pyme en Venezuela también tienen un origen remoto, vinculado al emprendimiento de la actividad económica. El reconocimiento y la individualización de los establecimientos socioeconómicos de carácter productivo y comercial denominados pyme resultan de un esfuerzo sostenido de diversos actores sociales para estructurar la economía nacional. Con esto, no solo se busca promover y garantizar el ejercicio de los derechos económicos, sino también distinguir las formas de negocio, a efectos de focalizar la atención y promoción pública.

De acuerdo con Luchena Mozo (2007), con el desarrollo del Derecho Positivo, fueron promulgados los Códigos de Comercio, en los cuales se contemplaron y regularon las empresas como figuras jurídicas. Según el Código de Comercio de Venezuela (Congreso de Venezuela, 1955), las pyme podían constituirse como las siguientes figuras jurídicas: 1) compañías de responsabilidad limitada; 2) sociedades en comandita, y 3) sociedades en nombre colectivo.
Más adelante, también podían ser compañías anónimas.

En las tres primeras formas asociativas enunciadas, el Legislador contempló el espíritu de confianza e intimidad presente entre los socios de una pequeña y mediana empresa, así como su libertad para definir el funcionamiento y la operatividad de la sociedad. En el caso de la compañía anónima, la regulación es más estricta y supone una vinculación de carácter comercial entre los socios.

La inclusión de la figura de las pyme en el Derecho contemporáneo se ha hecho con el propósito de promover la continuidad y el desarrollo de estos establecimientos, en consideración de la relevancia que tienen para proveer seguridad y bienestar a la sociedad. De acuerdo con Luchena Mozo (2007), la distinción legal de las pyme tiene ventajas frente a las grandes empresas, entre las que se destacan: 1) fuentes de financiación con tasas preferenciales; 2) exenciones fiscales; 3) plazos de amortización flexibles; 4) estímulos para la exportación; 5) ofertas de formación para sus recursos humanos, y 6) acceso a bienes de producción subvencionados o donados.

Según las estadísticas del Instituto Nacional de Estadística de la República Bolivariana de Venezuela 
(2010), en este país los establecimientos tipo pyme abarcan un $91 \%$ del tejido empresarial formalmente constituido y proveen empleo a más de $41 \%$ de la población activa. Por lo anterior, su rol en esta Nación, al igual que en la mayoría de países del mundo, no solo es decisivo para el porvenir económico, sino también para el desarrollo humano y la calidad de vida de los habitantes.

Aunque en la Ley Orgánica del trabajo, los trabajadores y las trabajadoras (Presidencia de la República, 2012) se incorporan algunas definiciones relacionadas con los entes empresariales industriales y comerciales en la República de Venezuela, no se hace una distinción entre pequeñas, medianas y grandes empresas. Sin embargo, en el Artículo 5 del Decreto con rango, valor y fuerza de ley para la promoción y el desarrollo de la pequeña y mediana industria y unidades de propiedad social (Presidencia de la República, 2008), se contemplan las definiciones correspondientes a la pequeña y mediana industria. En este mismo texto se definen los siguientes criterios para distinguirlas:

Pequeña y Mediana Industria: toda unidad organizadajurídicamente con la finalidad de desarrollar un modelo económico productivo mediante actividades de transformación de materias primas en insumos, en bienes industriales elaborados o semielaborados, dirigidas a satisfacer las necesidades de la comunidad.

Se considerará Pequeña Industria a aquellas que tengan una nómina promedio anual de hasta cincuenta (50) trabajadores y con una facturación anual de hasta cien mil Unidades Tributarias (100.000 UT).

Se considerará Mediana Industria a aquéllas que tengan una nómina promedio anual de hasta cien (100) trabajadoresy con una facturación anual de hasta doscientas cincuenta mil Unidades Tributarias (250.000 UT). Tanto en la pequeña como en la Mediana Industria ambos parámetros son recurrentes (pp. 6-7).

La referencia legal venezolana sobre los establecimientos de tipo pyme solo comprende a las unidades económico-productivas involucradas en procesos de transformación y fabricación de productos, es decir, las pertenecientes al sector industrial. En relación con una definición para las empresas dedicadas al comercio de mercancías y a la prestación de servicios, el Legislador ha quedado en deuda, lo que ha revelado su voluntad de privilegiar al sector industrial secundario frente al empresarial terciario. No obstante, esta definición legal para las pequeñas y medianas industrias es extensiva, por analogía, a las pyme no industriales.

De algún modo, los establecimientos clasificables como pyme exhiben 
ciertas características comunes en todo el planeta, pero su naturaleza filosófica varía en atención al contexto en donde se establecen y operan. Esto sucede, entre otros aspectos, en función de los factores culturales, económicos, legales, sociales y tecnológicos propios de cadaregión. Por esta razón, una definición de pyme debería atender a la esencia de esta organización, sin restringirse a estándares económicos, ya que estos entes productivos manifiestan un grado de complejidad propio que debe interpretarse con criterios tanto cuantitativos como cualitativos.

Según Cipolla (2002), desde el siglo XIX se aprecia el alto grado de especialización exhibido por muchas unidades de producción y comercialización susceptibles de ser consideradas como pyme. En estos negocios, dirigidos con frecuencia por profesionales con formación universitaria y técnicos empíricos, se adopta un modelo de gerencia conforme a los postulados de autores clásicos de la administración como Taylor (2003) y Fayol (1987), entre otros.

De acuerdo con Johnson, Scholes y Whittington (2006), la pequeña y mediana empresa presentan dificultades para acceder a múltiples tipos de recursos, en especial, a los financieros y tecnológicos; sin embargo, su gerencia suele adaptarse a estas restricciones mediante la optimización de los escasos recursos disponibles. Esto, en algunos casos, puede conducirla a obtener una mayor efectividad en el aprovechamiento de los mismos, que aquella que lograría alcanzar una gran empresa.

Según Navas y Guerras (2001), entre las características distintivas de las pyme en el siglo XXI está la flexibilidad, fundamentada en la simplicidad de su estructura organizativa y su mayor proximidad al cliente. Un aspecto determinante para propiciar la flexibilidad en la organización de las pyme se deriva de los estrechos vínculos entre los integrantes de la empresa. Estos, por lo general, son de tipo familiar, lo cual facilita el logro de acuerdos y compromisos, sin que se requieran prolongadas negociaciones. Esto permite acometer con más rapidez los cambios precisos, conforme al criterio de la dirección en la empresa.

La flexibilidad en las pyme no siempre está implícita, pero puede apreciarse como potencialmente superior, en comparación con la existente en las grandes empresas. En ellas, el gerente-propietario puede estar presente en las negociaciones y operaciones de compra y venta y adecuar con sencillez y de manera directa los procedimientos y las políticas organizacionales, en provecho 
de sus clientes y de la organización, aunque no suceda en todas las ocasiones. El gerente de una pequeña o mediana empresa también puede negociar de modo estricto conforme a parámetros recurrentes, rutinarios e inmutables. En este caso, desaprovecha la oportunidad de emplear el recurso de la flexibilidad como una fuente de ventaja competitiva.

Pese a que la estructura de las pyme suele ser simple y horizontal, la figura del gerente tiende a concentrar el poder para dirigir la organización e incluso decide acerca de los aspectos más elementales, los cuales, en las condiciones ideales, podrían ser delegados a otros miembros del equipo. Si la empresa es de tipo familiar, el gerente podría incurrir en el error de mezclar su rol de jefe de familia con el de directivo. En estos casos, su autoridad será incuestionable y tendrá la última palabra para decidir si flexibiliza o no su organización y si la ajusta a los requerimientos y las necesidades de sus clientes.

Navas y Guerras (2001) sostienen que hay cada vez un número mayor de pyme caracterizadas por su alta especialización y conformadas por profesionales calificados, dedicadas a la producción de bienes y a la prestación de servicios de considerable valor tecnológico. En Venezuela, la generación de tecnología por parte del sector pyme es más bien modesta. Sus esfuerzos por comprender las tecnologías recientes y aplicarlas en la prestación de servicios son destacados, no solo para tratar de satisfacer las necesidades de sus clientes individuales, sino también las de las grandes empresas con las que mantienen una relación comercial.

\subsection{Gestión estratégica aplicable a las pyme venezolanas}

Las expectativas relacionadas con la implementación de la administración estratégica en las empresas se refirieron, en principio, a la búsqueda de certidumbre y orden en medio de un contexto dinámico y en permanente evolución. Se consideraba que la certidumbre se alcanzaría por medio del reconocimiento, del monitoreo y de la valoración de las variables más determinantes para el desempeño de la empresa. El orden, por su parte, se fundamentaba en las estrategias concebidas como una serie de acciones metódicas, diseñadas a partir del adecuado reconocimiento del entorno.

La formulación de la estrategia, entendida como un sistema de administración de empresas, registra tres generaciones que se desarrollaron desde la década del sesenta. De acuerdo con Porter (2005), hubo una coexistencia entre la primera y 
la segunda generación entre 1960 y 1980. En la primera se contempló un sistema de administración basado en la planificación estratégica. Comprendía todas las actividades de la empresa y atendía al pensamiento estratégico diseñado por la dirección de la organización. Se sustentaba en la consideración de que era necesario fijar planes estables a largo plazo, orientados a posibilitar el crecimiento de la organización, en armonía con ciertos valores como el servicio al cliente, la orientación al mercado y el desarrollo del recurso humano.

Según Fernández Rodríguez (1987), a mediados de 1970 comenzó a surgir la segunda generación en el discurso de la gestión estratégica, impulsado por el importante incremento que se presentó en la complejidad del entorno. Podría indicarse que aquí emerge el principio dialógico planteado por Morin (2002), conforme al cual dos términos pueden ser complementarios y antagonistas. Este sería el caso del orden y el desorden de los mercados. Porter (2005) afirma que en la segunda generación se desarrolla una administración estratégica, en sustitución de la planificación estratégica. Las empresas empiezan a considerar las declaraciones de las misiones de los negocios como fundamento básico de una gestión dispuesta a efectuarse con base en planes estratégicos a corto, mediano y largo plazo.
En esta generación de la administración estratégica, el gerente se integra con más amplitud y decisión al fenómeno del direccionamiento de la empresa. Este requiere, según Damiani (2009), entender y comprender la realidad para tratar de construirla. Como elementos distintivos de esta generación, se destacan la consideración del sistema de valores y el interés por generar una cultura organizacional específica y útil a los propósitos de la compañía. La administración estratégica se plantea para posibilitar la continuidad de la organización como fin último, en atención a una vocación de servicio y para tratar de responder socialmente ante las comunidades, mientras se ejerce una conciencia ecológica y se proyecta solidaridad y dedicación al cliente.

Entre 1975 y 1985, apareció la tercera generación de la formulación estratégica. Para Porter (2005), esta comprendió una dirección o conducción de la organización conforme a problemas estratégicos, lo que implica la adopción de medidas rápidas y flexibles, dispuestas para incrementar la generación de valor en cada actividad de la compañía e identificar las ventajas competitivas y las claves del éxito en la gestión empresarial. En esta generación, el fin de la gestión estratégica es la continuación de un esfuerzo soste- 
nido para viabilizar el negocio. Para esto, se incrementa el compromiso con los clientes y las comunidades y se trata de exhibir cierta lealtad hacia ellos y de ampliar los campos de acción en el sistema socioeconómico por medio del cumplimiento de la responsabilidad social.

En el modelo formal-analítico, propio de la estrategia organizacional, esta puede ser estructurada en dimensiones de acuerdo con el tamaño de la empresa. Así, es posible esbozar un proceso de formulación estratégica tanto para la gran empresa como para la pequeña y la mediana.

Para implementar el mencionado modelo formal-analítico de administración estratégica en una pequeña o mediana empresa, en un contexto como el venezolano, se requiere llevar a cabo una serie de actividades. La primera es la definición de la misión empresarial, manifestada en una expresión escrita o verbal de las ambiciones de la empresa y de su propósito en la colectividad. En el contenido de la misión se destaca un elemento subjetivo relativo a la cultura, al objetivo y a la identidad de la organización. Asimismo, se incorpora un aspecto objetivo, concerniente a la situación actual y futura de la compañía, sus campos de negocio y sus capacidades presentes y potenciales.
En las pyme venezolanas que cuentan con estructuras familiares, la misión del negocio suele ser expresada verbalmente y desarrollada de manera unilateral por parte del gerente. En el contenido de la misión, es usual adoptar la cultura e identidad de la familia, en relación con la dimensión subjetiva, junto con un fin de la organización fundamentado en la visión del emprendedor.

El elemento objetivo de la misión también es definido por las ambiciones de la familia entendida como un grupo, en especial, cuando se trata de contemplar la situación futura de la empresa. La identificación de las capacidades presentes y potenciales de la compañía, como fundamento de las estrategias a formular, se basa en la experiencia del gerente y de los demás miembros de la organización. Sin embargo, suele recurrirse también al uso de métodos objetivos para identificar con más certeza dichas capacidades, cuando el objeto de la empresa implica la ejecución de actividades medibles en cada una de sus fases.

En el caso de las pyme no familiares, el proceso de definir la misión suele estar dirigido y encargado por el propietario. Si este no ejerce la gerencia del negocio, la responsabilidad recae sobre el gerente, quien está sujeto a atender las directrices 
del propietario. El proceso para el establecimiento de la misión puede ser participativo e involucrar a los miembros de la organización. En la pyme no familiar, la cultura está influida por los valores del gerente, quien, día a día, deja testimonio a los miembros de la organización mediante su proceder. El aspecto objetivo de la misión, en este tipo de pyme, está definido por el gerente o fundador de la empresa; no obstante, la previsión de la situación futura de la compañía es determinada por el propietario de la organización desde su creación.

Una vez definida e identificada la misión empresarial, se procede a establecer el atractivo de la industria o del sector comercial y a fijar la posición competitiva del negocio. Estas actividades, incluidas en la segunda fase del proceso de gestión estratégica, requieren considerar la información existente en diversas fuentes, algunas onerosas y otras gratuitas. En principio, es usual que una pequeña o mediana empresa no disponga de los recursos financieros necesarios para contratar los servicios de una firma de consultoría o una organización especializada en análisis industriales y de mercados. Por esta razón, la experiencia del gerente y la información gratuita disponible, como la existente en periódicos, revistas, reportes de instituciones pú- blicas y los informes ofrecidos por gremios empresariales e industriales, son las fuentes más utilizadas en estas fases del proceso de gestión estratégica.

Luego de redactar la misión, determinar el atractivo de la industria y fijar la posición competitiva de la empresa, se hace un análisis de debilidades, amenazas, fortalezas y oportunidades (DAFO), para determinar estos aspectos internos (DF) y externos (AO) propios del negocio. La identificación de las amenazas y oportunidades tiene lugar a partir de una revisión del entorno, trascendente al sector industrial o comercial, enfocado en aspectos clave de la naturaleza comercial de las actividades de la empresa.

\subsection{La responsabilidad social en las pyme venezolanas}

La RSE puede asumirse como el compromiso de los empresarios para contribuir al desarrollo social y económico de la comunidad, mediante la facilitación de un aporte superior al implícito en el desarrollo de su actividad industrial o comercial. Este concepto está relacionado con el ejercicio de una actividad empresarial para garantizar la sustentabilidad del ambiente a mediano y largo plazo, al restringir, si es el caso, los márgenes de ganancia, en provecho 
del bienestar de las generaciones futuras.

La implementación de la RSE está orientada a impactar de modo favorable en la comunidad tanto interna como externa, en un esfuerzo por mejorar las condiciones del entorno, sin enfocarse en soluciones inmediatas o aisladas. El compromiso de la gerencia, implícito en este tipo de responsabilidad, trasciende a la cúpula administrativa de la empresa y, por esta razón, no es asociable con un estilo específico de gestión.

La RSE no es homologable a una política filantrópica. Mientras esta última obedece a la solidaridad requerida, la responsabilidad social estructura planes concretos ejecutados con el fin de proveer desarrollo y bienestar, sin que para esto se necesite una solicitud previa por parte de terceros.

Como fundamento filosófico de la responsabilidad empresarial, se destaca la ética manifestada por los propietariosy directivos de las empresas. Esta se vincula con el propósito de procurar una armonía entre la generación de riqueza y un desarrollo sostenible para las comunidades directa e indirectamente vinculadas con la organización o industria. El efecto de la RSE en la sociedad no solo está dispuesto para ser percibido por los clientes de la compañía, sino por todos sus stakeholders y por los competidores. Estos se benefician también de la buena imagen del sector industrial, promovida por determinadas empresas socialmente responsables.

Paralela al componente ético de la RSE se destaca la solidaridad. Esta es manifestada por el empresario como una adhesión a las grandes causas de la humanidad, como el mejoramiento de la calidad de vida, la reducción de la pobreza y la protección del ambiente, entre otras. Este valor revela el interés de la organización por disponer parte de sus recursos para destinarlos a acciones en provecho de aquellos que posibilitan la continuidad y el desarrollo de la empresa, en particular, desde el rol de clientes o usuarios de bienes y servicios prestados por esta. La manifestación de la solidaridad en el cumplimiento de la RSE no solo se manifiesta de modo reactivo ante las contingencias de la comunidad, sino también por medio de acciones de carácter preventivo, tendientes a preservar los bienes más significativos de la sociedad.

De acuerdo con Méndez Rivas (2004), el desarrollo de la RSE en Venezuela se aprecia desde el siglo XIX. En ese entonces, algunos empresarios hacían aportes a sus comu- 
nidades, no solo para contribuir caritativamente con el fin de enaltecer su apellido y cumplir con deberes de origen religioso, sino para implementar acciones sistematizadas, de carácter voluntario, que procuraban favorecer el desarrollo. Entre las iniciativas impulsadas por los empresarios de esa época se reconocen, entre otras, diversas colaboraciones en programas de alfabetización, sanidad y salubridad.

En Venezuela, la implementación de la RSE ha estado orientada a participar en la tarea gubernamental de reducir los desequilibrios sociales existentes en el país. Hasta finales del siglo XIX, este país tenía una economía dinamizada por el sector agrícola. Durante esta etapa económica, los recursos generados contribuyeron a consolidar el Estado venezolano, pero fueron insuficientes para llevar el progreso a todo el territorio nacional, en especial, a aquellas zonas rurales en donde se generaban los recursos mediante la explotación agrícola y pecuaria. Ante el acentuado desequilibrio social, algunos empresarios del sector primario emprendieron actividades relacionadas con sus labores productivas, tendientes a contribuir a la generación de bienestar para las familias relacionadas con el negocio.

Entre finales del siglo XIX y principios del XX, la economía venezo- lana se dinamizó con el inicio de la explotación petrolera. Este negocio facilitó al Estado venezolano una ingente cantidad de recursos, los cuales fueron oportunos para modernizar el territorio nacional, mientras se mejoraba la calidad de vida de sus habitantes. Los ingresos extraordinarios permitieron que el Estado extendiera sus políticas y planes de desarrollo; sin embargo, el desequilibrio social continuo, cada vez más acentuado en las zonas rurales carentes de petróleo para extraer, así como el desarrollo de la industria petrolera y otros negocios relacionados, causaron un impacto desfavorable en el entorno. Aunque el Gobierno intervino con la sanción de regulaciones, algunos empresarios contemplaron los hechos con atención $y$ decidieron ejecutar acciones tendientes a revertir las secuelas de los procesos industriales. Esto evidencia un comportamiento éticamente apropiado que puede ser interpretado como una referencia de RSE.

A mediados de 1950, algunos empresarios comenzaron a plantear iniciativas individuales y gremiales, para vincular la gestión de la empresa a la problemática social. La relación de estos actores con los desafíos y problemas de las comunidades no solo se limitó a la identificación e implementación de soluciones y alternativas, pues se materializó en asistencia y ayudas individuales y 
gremiales, tanto unilaterales como en vinculación directa con el Estado.

El primer precedente de una iniciativa gremial para el cumplimiento de la RSE fue tal vez la constitución, en 1964, de la asociación civil Dividendo Voluntario para la Comunidad (2012) por parte de un grupo de empresarios, cuyos fines fueron "difundir y propagar el principio de responsabilidad de la empresa privada en el desarrollo socio-económico del país, estimular la acción de los asociados, proporcionar información y dar a conocer la labor social que cumple la empresa privada" (DVC, 2012, p. 1).

El auge económico de Venezuela, a partir de 1960, propició el establecimiento de empresas transnacionales en el país que ejecutaron prácticas gerenciales concebidas en otras latitudes, comprometidas, entre otros aspectos, con la implementación de la RSE. Estas fueron consideradas no solo por el propósito de exhibir un comportamiento ético y solidario, sino también por el de proyectar una imagen favorable a la comunidad, que fuera provechosa para estimular, a mediano y largo plazo, la actitud del consumidor respecto a la marca de los productos ofrecidos.

En Venezuela, como sucede en el mundo, la implementación de la RSE ha estado asociada con las grandes empresas e industrias. Esto ha conducido a la denominación del discurso como "responsabilidad social corporativa". De esa forma, se expone como una manifestación común de la gestión llevada a cabo en las grandes corporaciones, posibilitada por una significativa disposición de recursos humanos y financieros. Si la responsabilidad social es contemplada en una gran empresa, su ejecución responderá a directrices centralizadas contenidas en el plan estratégico corporativo.

De acuerdo con lo indicado en el primer aparte de este artículo, las pyme en Venezuela alcanzan un porcentaje superior a $91 \%$ del tejido empresarial. De este modo, si solo las grandes corporaciones procuraran ser socialmente responsables, el porcentaje de empresas comprometidas con la RSE en Venezuela solo podría ser de un $9 \%$. Por lo anterior, es pertinente preguntarse, como se hace en esta investigación, si las pyme venezolanas son gestionadas al margen de la RSE.

\section{METODOLOGÍA}

La metodología aplicada se basó en una perspectiva fenomenológica de tipo cualitativo y fue elegida con el fin de profundizar en el fenómeno mediante la integración del investi- 
gador en el mismo. Dicho fenómeno corresponde a la implementación de la gestión estratégica en las pyme, en particular, al propósito de cumplir con la RSE.

El hecho de recurrir a la perspectiva cualitativa obedeció, de acuerdo con lo indicado por Husserl (1986), al carácter descriptivo implícito en este.

Junto con la fenomenología, se destaca el uso de la teoría comprensiva de Weber (1978) como otro gran soporte filosófico y teórico del actual paradigma cualitativo de la investigación científica. Esta teoría se correlaciona con el enfoque fenomenológico por el esfuerzo para acceder al conocimiento, en referencia a la perspectiva de los sujetos que perciben y experimentan las situaciones.

El método comprensivo se fundamenta en la interpretación; por consiguiente, antes de explicar un fenómeno, debe ser comprendido. En línea con lo indicado por Weber (1978), la interpretación permite cubrir las deficiencias implícitas en la simple explicación y abarcar un espectro más amplio, hasta despejar la mayor cantidad posible de incógnitas.

El desempeño de los individuos en sus tareas dentro de la organización exhibe elementos tanto cualitativos como cuantitativos. Los aspectos cualitativos se refieren a actitudes, valores, principios y costumbres que se manifiestan en el comportamiento organizacional.

En el seno de la organización tienen lugar muchos hechos y fenómenos. Desde la perspectiva cualitativa, el investigador puede reconstruir los hechos, al emplear su propia conciencia e interpretar lo observado con detenimiento, hasta ilustrar el fenómeno en profundidad, a diferencia de lo que puede alcanzar la explicación cuantitativa.

Como técnicas de investigación, se emplearon la observación participante y la entrevista en profundidad a los gerentes de doce organizaciones empresariales del tipo pyme que pertenecen al sector industrial, establecidas en el estado Táchira (Venezuela). Con la observación se recopiló, registró y seleccionó información visual y auditiva, con el fin de describir la gestión estratégica de la responsabilidad social llevada a cabo en esas pyme.

La situación apreciada fue la ejecución de la dirección empresarial enfocada en la estrategia y la responsabilidad social por parte del gerente de la empresa y de cualquier otro individuo con facultades de dirección o conducción equivalentes a las de 
este. El contexto de la observación correspondió a reuniones formales e informales del personal y de la Junta Directiva, durante las cuales se consultó a los individuos sobre su proceder en los aspectos vinculados con la investigación.

Las entrevistas en profundidad se implementaron para lograr la expresión libre y detallada del entrevistado acerca del tema, quien pormenorizó en sus opiniones respecto a la gestión estratégica y la responsabilidad social. De igual manera, explicó los procesos gerenciales relacionados con ambos conceptos en las empresas dirigidas.

\section{RESULTADOS}

De conformidad con la información recopilada, la estrategia es conocida por los gerentes como un instrumento de gestión y no como todo un campo de la gestión. En cuanto al empleo de la estrategia, no se estimaron procesos destinados a organizar su formulación e implementación; sin embargo, se identificó recurrencia a la estrategia de tipo emergente. Durante las entrevistas, se reconoció la importancia de definir la misión empresarial, pero no en todos los casos se vinculó con la gestión estratégica.

Aunque el fundamento filosófico de la RSE reposa sobre la ética y la solidaridad del empresario y su aplicación suele estar ligada con las grandes corporaciones, para un pequeño o mediano empresario es posible gestionar su negocio de forma socialmente responsable, a pesar de que carezca de cuantiosos recursos económicos para poder hacerlo. De acuerdo con Monsen (citado en Stoner \& Wankel, 1989), el activismo social de las empresas puede clasificarse en cuatro capas: el primero se verifica cuando el gerente le sirve con su gestión a la sociedad y obedece a la ley; el segundo trasciende al simple cumplimiento del ordenamiento jurídico y trata de satisfacer las expectativas del público, al considerar su opinión; el tercero constata la existencia de una previsión de las expectativas del público, y el cuarto conduce a que el activismo corresponda a la creación de nuevas expectativas para el público, vinculadas con el testimonio de un compromiso moral por parte de la dirección.

Según Vázquez Gallardo y Polo Castilla (2013), el grado de activismo social relativo al cumplimiento del ordenamiento jurídico es más bien obvio; no obstante, en América del Sur, esto parece todo un desafío. A partir de estimaciones de la Organización Internacional del Trabajo (citada en De Soto, 2002), se puede afirmar que, desde 1990, un $85 \%$ de 
los empleos en Sudamérica y el Caribe ha sido generado por establecimientos del sector informal, los cuales cuentan con estructuras similares a las que poseen las pyme legalmente constituidas. Entre los factores más críticos para el desarrollo del sector informal extralegal se destacan la corrupción, la burocracia y la falta de capital e incentivos para legalizar el ejercicio de la actividad comercial.

La Federación de Cámaras y Asociaciones de Comercio y Producción de Venezuela (2014) reporta una progresiva disminución de las pyme; sin embargo, en atención a lo indicado en el párrafo anterior, esta supuesta desaparición puede corresponder más bien a la conversión de pyme legales en establecimientos informales. Por consiguiente, la sujeción de la gerencia de la pequeña o mediana empresa al marco legal venezolano representa el punto de partida de una gerencia socialmente responsable.

En la gran empresa, la responsabilidad social puede estar dentro del programa estratégico corporativo, con el propósito de proyectar una gestión socialmente responsable de carácter integral, estimulada, según Torres (2006), por la necesidad de mejora continua de su imagen. En el caso de las pyme, esta responsabi- lidad debería estar inserta en el sistema de gestión estratégico. En las pyme investigadas, se encontró una vinculación de la responsabilidad social con los esfuerzos de la organización por conectarse con los clientes y el contexto en donde opera.

El punto de partida de la gestión estratégica formal en las pyme es la declaración de la misión empresarial y, por esto, el compromiso con la RSE debe estar reflejado en ella. La enunciación de los valores relativos a la ética y la solidaridad insinúan el desarrollo de una gestión socialmente responsable. A partir de esta, el reto de la gerencia en la pyme consiste en dejar testimonio de lo propuesto en la misión. En las misiones de algunas pyme investigadas, fue posible distinguir declaraciones dirigidas a mantener una operatividad en armonía con la sociedad.

De conformidad con los gerentes entrevistados, el compromiso ético y la solidaridad en la gestión del negocio se ponen en práctica con un desempeño gerencial ajustado al ordenamiento jurídico vigente, de manera que se inicia la gestión socialmente responsable en las pyme sustentada en la ética del gerente. Este comportamiento gerencial se adecuaría al primer grado de activismo social empresarial mencionado. 
Aunque este no es valorado por algunos autores como una muestra real de responsabilidad social, en América Latina y en particular en Venezuela, en donde el sector informal convive con el formal, esto dejaría claro un mensaje vital: la presencia de un obrar correcto por parte del empresario. Esto le permitiría, por consiguiente, alejarse de una economía extralegal impulsada con frecuencia por actividades ilícitas en un marco de injusticia social.

Una vez el empresario hace una gestión acorde con los preceptos legales, es posible impulsar una responsabilidad social adecuada a la segunda dimensión de activismo social empresarial, concerniente al cumplimiento de las expectativas del público en un grado superior a la sujeción legal. El desarrollo de una responsabilidad social por parte del gerente de pyme, que obedece a este segundo grado de activismo social, requiere la colocación de recursos financieros y humanos para tal fin, los cuales, como se ha indicado, pueden escasear en esta categoría de empresas.

La creatividad y la suma de esfuerzos con las comunidades inmediatas de las pyme facilitan una gestión socialmente responsable. Pese a que en el programa de gestión estratégica de las pyme es factible formular estrategias tendientes a ocuparse del entorno, el cumplimiento de dicha responsabilidad suele limitarse a la comunidad inmediata del establecimiento; no obstante, la magnitud del activismo social puede transcender y magnificarse de acuerdo con la evolución y disponibilidad de los recursos con los que cuenta la pequeña o mediana empresa.

\section{CONCLUSIONES}

La gestión estratégica de empresas es uno de los más adecuados caminos existentes para la gestión de cualquier tipo de establecimiento empresarial o industrial, incluidos los de la categoría pyme. En el caso de las grandes empresas, las prácticas asociadas con la RSE son contempladas en los programas estratégicos corporativos, con el fin de integrarse solidariamente con la sociedad mientras se benefician de las ventajas de poseer una imagen favorable, estimables en materia de crecimiento y rentabilidad.

La RSE implica la necesidad de contar con los recursos para llevarla a cabo de manera efectiva. Las pyme también pueden desarrollar una gestión socialmente responsable, adecuada a sus dimensiones y capacidades y en relación directa con su contexto.

Es necesario que la disposición de sus gerentes para obrar en el marco de la responsabilidad social se fun- 
damente en una verdadera estrategia; de esta forma, podrán estimarse con mayor precisión el impacto de estas iniciativas sociales y su efecto a corto, mediano y largo plazo en el desempeño de la empresa.

\section{REFERENCIAS}

Cipolla, C. (2002). Historia económica de la Europa preindustrial. Barcelona: Crítica.

Congreso de Venezuela. (1955). Código de Comercio de Venezuela. Gaceta oficial de la República Bolivariana de Venezuela, número 475 del 21 de diciembre.

Damiani, L. (2009). Epistemología y ciencia en la modernidad. Caracas: Ediciones Faces.

De Soto, H. (2002). Los misterios del capital: por qué el capitalismo triunfa en Occidente y fracasa en el resto del mundo. Buenos Aires: Sudamericana S. A.

Dividendo Voluntario para la Comunidad [DVC]. (2012). Estatutos. Recuperado de http://www. dividendovoluntario.org/

Fayol, H. (1987). Administración industrial y general (10a ed.). Buenos Aires: El Ateneo.

Federación de Cámaras y Asociaciones de Comercio y Producción de Venezuela. (2014). Informe anual. Caracas: Autor.

Fernández Rodríguez, Z. (1987). Evolución del pensamento estratégico, Economistas, 28, 6-13.
Husserl, E. (1986). Ideas relativas a una fenomenología pura y una filosofia fenomenológica. México: Fondo de Cultura Económica. Instituto Nacional de Estadística de la República Bolivariana de Venezuela. (2010). Informe anual. Caracas: INERBV.

Johnson, G., Scholes, K. \& Whittington, R. (2006). Dirección estratégica (7a ed.). Madrid: Pearson.

Luchena Mozo, G. M. (2007). Fiscalidad de la empresa familiar. Barcelona: Atelier Libros.

Martín Martín, V. \& Cepeda González, I. (2006). Lecciones de Historia Económica. Madrid: McGraw-Hill.

Méndez Rivas, C. (2004). Responsabilidad social de empresarios y empresas en Venezuela durante el siglo $X X$. Caracas: Strategos consultores.

Morin, E. (2002). Introducción al pensamiento complejo. Madrid: Gedisa.

Navas, J. E. \& Guerras, L. A. (2001). La dirección estratégica de la empresa. Madrid: Civitas.

Porter, M. E. (2005). Estrategia y ventaja competitiva. Barcelona: Deusto.

Presidencia de la República. (2008). Decreto con rango, valor y fuerza de ley para la promoción y desarrollo de la pequeña y mediana industria y unidades de propiedad social, Gaceta Oficial, 1-34. Recuperado de http://images. 
eluniversal.com//2008/08/04/ leyh12.pdf

Presidencia de la República. (2012). Ley orgánica del trabajo, los trabajadores y las trabajadoras. Decreto $N^{o} 8.938$ del 30 de abril de 2012. Recuperado de http:// www.lottt.gob.ve/

Stoner, J.\& Wankel, C.(1989). Administración. México D. F.: Prentice-Hall Hispanoamericana S. A.

Taylor, F. W. (2003). Principles of Scientific Management. Londres: Routledge.
Torres, R. P. (2006). La responsabilidad social corporativa en las Pyme, Lan Harremanak, Revista derelaciones laborales, 14, 77-96.

Vázquez Gallardo, D. \& Polo Castilla, F. (2013). Diseño de un sistema de comunicación eficaz para la responsabilidad social en las pyme. Tribuna de Economía ICE, 872, 161-178.

Weber, M. (1978). Die protestantische Ethik und der Geist des Kapitalismus. Gütersloh: GTB Siebenstern. 